http://jmscr.igmpublication.org/home/ ISSN (e)-2347-176x ISSN (p) 2455-0450

crossref DOI: https://dx.doi.org/10.18535/jmscr/v7i12.93

Journal Of Medical Science And Clinical Research

\title{
A Comparative Study between Epidural Butorphanol, Nalbuphine, and Fentanyl for Post-Operative Analgesia in Lower Abdominal Surgeries: A Prospective Randomised Double Blinded Trial
}

\author{
Authors \\ Dr Bura Anil Sarath Chandra ${ }^{1}$, Dr B.Vishnu Mahesh Babu ${ }^{2 *}$ \\ ${ }^{1}$ M.D (Anaesthesia), Postgraduate in Department of Anaesthesia, Rangaraya Medical College, Kakinada \\ (A.P) \\ ${ }^{2}$ Associate Professor, Department of Anaesthesia, Rangaraya Medical College, Kakinada (A.P) \\ *Corresponding Author \\ Dr B.Vishnu Mahesh Babu M.D (Anaesthesia)
}

\begin{abstract}
Background: The popular technique nowadays regarding the management of acute postoperative pain is the use of epidural opioids.

Aim: To assess the postoperative analgesic benefits in patients who administered epidural Fentanyl, Nalbuphine, and Butorphanol as adjuvants with local anaesthetics postoperatively for surgeries done under epidural anaesthesia.

Methods: Total 90 patients were scheduled for surgeries of lower abdomen belonging to age groups 1965 years were divided randomly into 3 groups of 30 each. For all patients, surgeries were performed by Epidural technique using bupivacaine 0.5\%. The study drug was administered through the epidural catheter in the postoperative period. Group F received Fentanyl $100 \mu \mathrm{g}$; Group N received nalbuphine 10 $m g$ and Group B received Butorphanol $2 \mathrm{mg}$ along with $0.125 \%$ bupivacaine, which was diluted with normal saline to $10 \mathrm{ml}$ each. The onset of action, duration of analgesia, quality of analgesia, hemodynamic changes, and side effects - such as nausea, vomiting, pruritus, sedation, respiratory depression, and urinary retention - were recorded.

Results: In all three groups, the demographic data were readily comparable. When compared to other groups, the onset of sensory blockade was significantly earlier in Group F (Fentanyl). Significantly, longer duration was observed in Group B (Butorphanol). There were no significant haemodynamic side effects observed in any of the groups.

Conclusion: Onset of analgesia is faster with Fentanyl, but pruritus is the ocassional side effect. Duration of analgesia is significantly prolonged with epidurally administered Butorphanol than that of epidural nalbuphine and epidural Fentanyl.

Keywords: Epidural analgesia, Fentanyl, Nalbuphine, and Butorphanol.
\end{abstract}

\section{Introduction}

The popular technique for post-operative pain management is administration of analgesics through the epidural route because it can be used alone or in combination with general anesthesia ${ }^{[1]}$. Administration of analgesics via epidural route decreases stress response to surgery and pain, minimizes the need for systemic analgesics, and 
facilitates early rehabilitation ${ }^{[2]}$. Epidural analgesia with local anesthetics was found to be effective in pain management ${ }^{[3]}$. Opioids were used as an adjuvant to local anesthetics and this combination was found to be synergistic ${ }^{[3]}$. Better pain relief, motor sparing and reduced toxicity were the benefits of this combination.

Fentanyl, a $\mu$ opiate receptor agonist, has analgesic potency greater than morphine. When compared to morphine and pethidine it has shorter duration of action and lesser respiratory depressant effect ${ }^{[4]}$.

Nalbuphine is a synthetically derived agonistantagonist opioid analgesic which is equal in potency as an analgesic to morphine and is about one-fourth potent as nalorphine as an antagonist. It has a ceiling effect on respiratory depression. Sedation is commonly associated with it when used in post-operative period as an analgesic ${ }^{[3]}$.

Butorphanol is also synthetically derived agonistantagonist opioid analgesic. It is an agonist on $\mathrm{K}$ receptor and either antagonist or partially agonist on $\mu$ receptor. It is considered safer than pure agonist opioids because of its ceiling effect on respiratory depression, lower addiction potential, lesser side effects like nausea, vomiting, pruritus, and urinary retention. It produces sedation more than that of morphine, which is desired in postoperative period ${ }^{[4]}$.
This study was done to evaluate and compare the post-operative analgesic benefits in patients administered epidural butorphanol, nalbuphine, and fentanyl as adjuvants with local anesthetics postoperatively for surgeries under epidural anesthesia and to compare their post-operative efficacy with respect to increase in duration of analgesia, reduction in total requirements of analgesics postoperatively and study side effects and complications, if any attributable to these drugs.

\section{Methods}

After obtaining approval from the institutional Ethical Committee of Rangaraya Medical college and Government General Hospital, Kakinada and after taking both written and informed consent, a total of 90 hospital inpatients belonging to age 19 to 65 years of either sex who were scheduled for surgeries of lower abdomen were selected for the study and were randomly divided to 30 in each group. This study conducted from June 2019 to September 2019.

\section{Type of study}

This is a prospective randomized double-blind study.

\section{Inclusion and Exclusion Criteria}

\begin{tabular}{|l|l|}
\hline \multicolumn{1}{|c|}{ Inclusion criteria } & \multicolumn{1}{c|}{ Exclusion criteria } \\
\hline 1. ASA I and II patients. & 1. Pregnant patients \\
2.Surgeries of the lower abdomen. & 2. Breastfeeding patients \\
3.Patients of age 19 to 65 years of either sex had & 3. ASA III and IV patients \\
no clinically significant cardiovascular or central & 4. Local infection \\
nervous system diseases. & 5. Known allergy to study drugs \\
& 6. Coagulopathies \\
& 7. Vertebral anomalies \\
& 8. Neurological diseases \\
& 9. Spinal level blockade above T6 \\
& 10. Renal insufficiency \\
& 11. Peptic ulcer disease \\
& 12. History of drug abuse \\
& 13. Patients in whom epidural anesthesia was not \\
& adequate and supplemented with other types of \\
& anesthesia. \\
& 14. Morbid obese patients \\
\hline
\end{tabular}


Patients were randomly divided into three groups

Group F ------- Fentanyl group

Group N ------- nalbuphine group

Group B -------- Butorphanol group

\section{Pre-anesthetic Evaluation}

Complete clinical history was taken, general and systemic examinations were done in detail. Routine laboratory investigations such as complete hemogram, bleeding time, clotting time, blood sugar, blood urea, serum creatinine and urine analysis, electrocardiography (ECG), and chest X-ray were carried out in all patients.

The patients were explained about the epidural technique with catheter in situ and its advantages and disadvantages. They were also educated about the usage of linear visual analog scale (VAS) for assessment of the intensity of post-operative pain and were instructed to mark on the scale at the point which he/she felt was representative of their level of discomfort.

\section{Premedication}

All patients were given with Tablet Alprazolam $0.25 \mathrm{mg}$ on the night before the surgery and were also kept nil orally for $6 \mathrm{hrs}$ before surgery.

\section{Anesthesia}

Epidural technique was adopted for surgery of the lower abdomen for all patients with $0.5 \%$ bupivacaine. The patient was made to lie supine on the operation table. Routine monitors such as ECG, noninvasive blood pressure (NIBP), and pulse oximetry were connected for every case, and base line readings were recorded. An intravenous line was secured with $18 \mathrm{G}$ canula and infusion Ringer lactate was started before starting the epidural technique. Drugs and equipment necessary for resuscitation and general anesthesia administration were kept ready.

An autoclaved epidural tray was used. After placing the patient either in sitting or lateral position under aseptic precautions, a skin wheal was raised at L2-L3 or L3-L4 interspace with $2 \mathrm{ml}$ of $2 \%$ lignocaine. The epidural space was identified using $18 \mathrm{G}$ disposable Tuohy needle with loss of resistance technique. Then, $20 \mathrm{G}$ catheter was passed through the epidural needle till about 2-3 cm of the catheter was in the space. The needle was withdrawn keeping the inserted epidural catheter in situ and was fixed to the back using adhesive tape. $3 \mathrm{ml}$ of $2 \%$ lignocaine with adrenaline 1:2,00,000 was injected through the catheter as a test dose and observed for any untoward reactions including drug interactions as well as intravascular or intrathecal injection.

After confirming correct placement of the catheter, epidural anesthesia was activated using $16-18 \mathrm{ml}$ bolus dose of $0.5 \%$ bupivacaine. Subsequent top up doses were given depending on the duration of surgery and intensity of pain. No narcotics were administered throughout the intraoperative period.

\section{Fluid Management}

With crystalloids and colloids. Blood was transfused only when indicated.

The following observations were made.

\section{Intraoperative}

- Onset of analgesia.

- Level of sensory blockade (maximum sensory level after 30 minutes).

- Monitoring BP (NIBP), Heart rate (HR), Respiratory rate (RR) and $\mathrm{SpO} 2$.

Duration of surgery was also noted.

- Onset of analgesia (sensory block): The time interval between administrations of local anesthetic $(0.5 \%$ bupivacaine $)$ epidurally to the loss of pinprick sensation at the site of surgical incision.

- Level of sensory blockade: The maximum sensory dermatome level after 30 minutes of administering the local anesthetic $(0.5 \%$ bupivacaine) in the epidural space. The local anesthetics usually get fixed to their respective receptors by 20 minutes, and regression of two dermatomes usually occurs after 30 minutes.

During intraoperative period, NIBP, HR, RR, and $\mathrm{SpO} 2$ were recorded before activating epidural 
anesthesia and subsequently at every 5 minutes till the end of the surgery.

After the surgery, the patients were shifted to recovery room and monitoring was continued. They were shifted to post-operative ward after recovery from motor blockade.

\section{Post-operative Period}

In the post-operative period, when the patients first complained of pain, intensity of pain was assessed using VAS scale. When the VAS score was $>5$, study drug was given through epidural catheter after confirming its proper position as:

- Group B - Received butorphanol $2 \mathrm{mg}$ with $0.125 \%$ bupivacaine diluted to $10 \mathrm{ml}$ in normal saline.

- Group F - Received fentanyl $100 \mu \mathrm{g}$ with $0.125 \%$ bupivacaine diluted to $10 \mathrm{ml}$ in normal saline.

- Group N - Received nalbuphine $10 \mathrm{mg}$ with $0.125 \%$ bupivacaine diluted to $10 \mathrm{ml}$ in normal saline.

The intensity of pain and pain relief was assessed using VAS at 5,10,15,30,60 minutes and thereafter hourly for $8 \mathrm{hrs}$ and then at $4 \mathrm{hrs}$ interval for $24 \mathrm{hrs}$ postoperatively. As and when the patient complained of pain during the period of observation, intensity of pain was assessed again using VAS to know the effect of the study drug given earlier. If it was $>5$, an intramuscular non-opioid analgesic as per the institutionally approved protocol was given.

VAS consisted of a $10 \mathrm{~cm}$ line, marked at $1 \mathrm{~cm}$ each on which the patient makes a mark on the line that represents the intensity of pain he/she was experiencing. Mark " 0 " represents no pain and mark " 10 " represents worst possible pain. The numbers marked by the patient was taken as units of pain intensity.

\begin{tabular}{|l|c|}
\hline VAS score & Intensity of pain \\
\hline $0-2$ & No pain to slight pain \\
$2-5$ & Mild pain \\
$5-7$ & Moderate pain \\
$7-9$ & Severe pain \\
10 & Worst possible pain (intolerable) \\
\hline
\end{tabular}

Following observations were recorded:

1. Onset of analgesia

2. Duration of analgesia

3. Quality of analgesia

4. Cardio- respiratory effects: HR, BP and RR were

5. Side effects such as sedation, pruritis, nausea, vomiting, respiratory depression and urinary retention, and hypotension.

\section{Onset of Analgesia}

The time interval from administration of the study drug (VAS score of $>5$ ) till VAS score became $<5$.

\section{Duration of Analgesia}

The time interval between onset of analgesia (VAS score <5), till patient complained of pain (VAS score >5) when rescue medication was administered.

Quality of analgesia was assessed during the duration of analgesia using pain score and compared between all the three groups.

\section{Quality of Analgesia}

\begin{tabular}{|l|c|}
\hline Pain score & Pain relief \\
\hline 0 & No pain relief \\
1 & Poor pain relief \\
2 & Fair pain relief \\
3 & Good pain relief \\
4 & Excellent pain relief \\
\hline
\end{tabular}

Bradycardia- A fall of HR by $20 \%$ from the basal HR.

Hypotension - Defined as a fall of systolic BP by $20 \%$ from basal systolic BP.

Respiratory depression - Bradypnea appears to be a more reliable clinical sign of early respiratory depression and a $\mathrm{RR}<10$ breaths/minutes was recorded as respiratory depression.

Side effects:

Sedation - quality of sedation after administering the study drug was based on sedation scoring.

- Grade 0 - No sedation, patient awake.

- Grade 1 - Mild sedation, patient awake but drowsy.

- Grade 2 - Moderate sedation, sleepy but arousable.

- Grade 3 - Severe sedation, unarousable. 
Other side effects that were observed and compared were pruritus, nausea, and vomiting.

\section{Statistical Analysis}

All data recorded and analysed using SPSS version 17 (statistical package for social sciences). Data were expressed as mean with a standard deviation. Discreet data were expressed as frequency with percentage of total. ANOVA with post hoc test was used to compare continuous variables. $\mathrm{p}<0.05$ was considered as significant.

\section{Results}

All the three groups were comparable in terms of age, sex and weight, duration and type of surgery (Tables 1 and 2).

Table 1: Demographic Data

\begin{tabular}{|l|c|c|c|}
\hline & GROUP B & GROUP F & GROUP N \\
\hline Age in years & $41.00+/-11.835$ & $41.87+/-12.280$ & $43.50+/-12.378$ \\
$\begin{array}{l}\text { Sex (female: } \\
\text { male) }\end{array}$ & $18: 12$ & $15: 15$ & $14: 16$ \\
$\begin{array}{l}\text { Weight (kg) of } \\
\begin{array}{l}\text { Duration on } \\
\text { surgery (minutes) }\end{array}\end{array}$ & $64.23+/-8.569$ & $61.30+/-7.702$ & $61.77+/-8.545$ \\
\hline
\end{tabular}

Table 2: Block Characteristics

\begin{tabular}{|c|c|c|c|}
\hline Time (minutes) & GROUP B & GROUP F & GROUP N \\
\hline $\begin{array}{ll}\text { Onset } & \text { of } \\
\text { analgesia } & \end{array}$ & $13.93+/-2.586$ & $7.808+/-1.606$ & $16.53+/-2.013$ \\
\hline $\begin{array}{l}\text { Level } \\
\text { analgesia }\end{array}$ & T8 & T8 & T8 \\
\hline $\begin{array}{l}\text { Duration of } \\
\text { analgesia } \\
\text { (minutes) }\end{array}$ & $479.17+/-43.279$ & $\begin{array}{c}198.10+/- \\
20.263\end{array}$ & $\begin{array}{c}313.50+/- \\
36.736\end{array}$ \\
\hline
\end{tabular}

\section{Onset of Analgesia}

The Mean time of onset of analgesia was 13.93 minutes, 7.80 minutes, and 16.53 minutes in Groups B,F and $\mathrm{N}$ respectively. Statistical analysis showed faster onset of analgesia in fentanyl group when compared to other two groups $(\mathrm{p}<0.05)$.

\section{Duration of Analgesia}

The mean duration of analgesia was 479.17 minutes in Group B, 198.10 minutes in Group F and 313.50 minutes in Group N. In butorphanol group the duration was significantly longer.

\section{Hemodynamic Changes}

In all the groups Blood pressure, Pulse rate, Spo2 and Respiratory Rate were monitored and were stable.

\section{Comparison of Mean Pain Score}

The mean pain score recorded was significantly lower in Groups B and F than in Group N.

All the patients in Groups $\mathrm{F}$ and $\mathrm{N}$ required analgesic supplementation within first 4-6 hrs and 6-8 hrs, respectively. Whereas, 5 patients of Group B required supplementation within 6-8 hrs, 8 patients between 8 and 10 hrs.

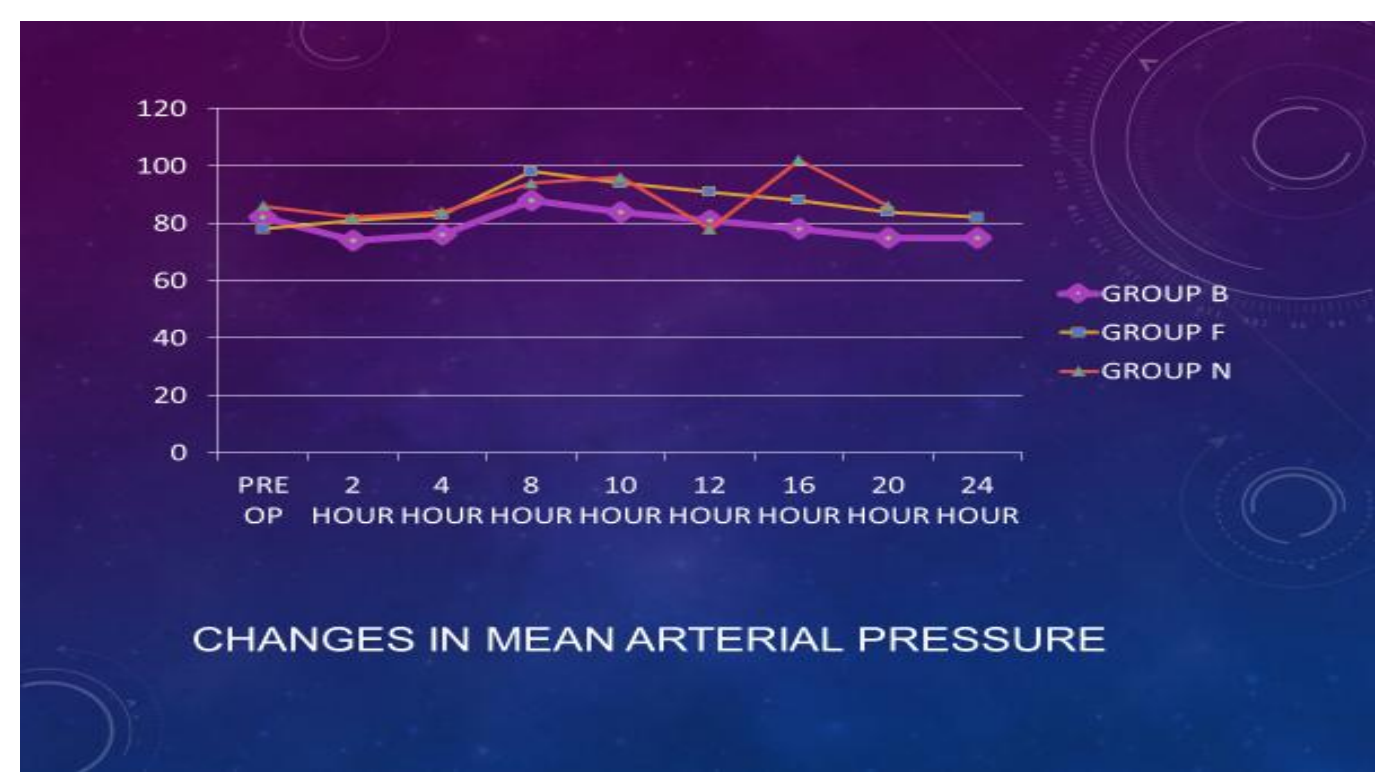



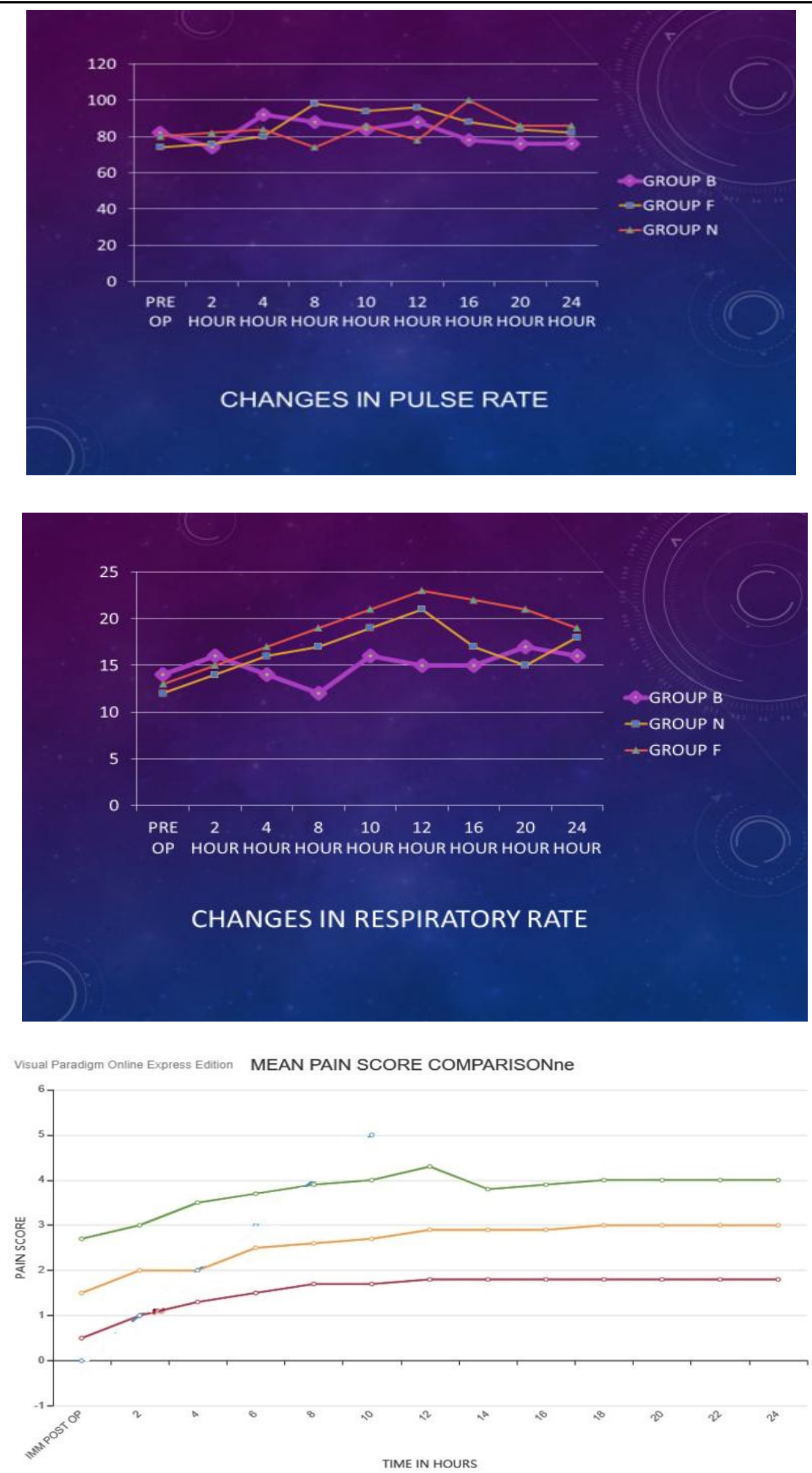

- - PAIN SCORE -O-GROUP B - - GROUP F - - - GROUP N 


\section{Complications}

Nausea and vomiting

Table 3 shows, in this study, $26.3 \%$ patients in Group B, 26.3\% patients in Group F and 47.4\% patients in Group $\mathrm{N}$ had nausea and vomiting. The fact that opioids and pain themselves cause vomitting and relatively high female proportion in the study group may be the reasons underlying.

Sedation

This was the main side effect seen in the butorphanol group which constituted $40 \%$ and
$3.3 \%$ of patients in the fentanyl group had sedation. Majority of the patients had mild sedation i.e, patient awake but drowsy which was statistically significant $(\mathrm{p}<0.001)$ when compared to that of the nalbuphine group.

Pruritus

None of the patients in nalbuphine group and butorphanol group had pruritus whereas three patients in fentanyl group had pruritus. Interaction with trigeminal nucleus in medulla is the cause of Pruritus induced by epidural opioids.

Table 3: Complications

\begin{tabular}{|l|c|c|c|c|}
\hline \multicolumn{1}{|c|}{ Complication } & Group B (\%) & Group F (\%) & Group N (\%) & p value \\
\hline Nausea \& vomiting & $5(26.3 \%)$ & $5(26.3 \%)$ & $9(47.4 \%)$ & 0.344 \\
Urinary retention & 0 & 0 & 0 & ------ \\
Respiratory & 0 & 0 & 0 & ---- \\
depression Sedation & $12(40 \%)$ & $1(3.3 \%)$ & $0(0 \%)$ & $\mathrm{p}<0.001$ \\
Pruritis & 0 & 3 & 0 & 0.045 \\
\hline
\end{tabular}

\section{Discussion}

Post-operative pain is acute pain, which starts with the surgical trauma and usually ends with tissue healing. It decreases with time after surgery and usually responds to analgesics. The effective pain relief is essential to the patients undergoing surgery and is very important both on humanitarian grounds and also in reducing postoperative morbidity, hence should be duly imparted by the treating anesthesiologist.

Severe pain leads to splinting, with resultant atelectasis and hypoxia. Besides this, poor control of pain may result in increased catecholamine secretion, which may in turn increase myocardial oxygen demand. Many studies have proved that improved post-operative analgesia can reduce the incidence of cardiac and pulmonary morbidity and mortality in patients undergoing major abdominal surgery.

The discovery of opioid receptors in the spinal cord enable us to clearly understand the action of narcotics through opioid receptors. kappa, one of the opioid receptors, is mainly involved in mediating the visceral pain.

The use of epidural opioids for management of acute post-operative pain had become an increasingly popular technique in recent times.
However, narcotics are associated with some disadvantages as they are not always simple to use and may be associated with some unpleasant adverse effects such as nausea and vomiting (postoperative nausea and vomiting), pruritus, urinary retention and respiratory depression.

spinal opiate receptors (kappa, к) stimulation, produce spinal analgesia but with fewer side effects. Therefore, a drug such as butorphanol, a mixed narcotic agonist/antagonist, acts as a mu $(\mu)$ agonist/antagonist and kappa agonist, also produces analgesia, associated with fewer side effects and also low abuse potential. Its high lipid solubility and high affinity for opioid receptors are additional factors which contribute to paucity of side effects with its use.

Nalbuphine is an agonist - antagonist, equipotent to morphine also has a low abuse potential. It is known to produce profound analgesia and is known to be associated with side effects like sedation. It commonly finds its place in clinical practice as it has a ceiling effect on respiratory depression.

Fentanyl was chosen for the study because of several advantages such as stable in salt solutions for more than $72 \mathrm{hrs}$, no neurolytic preservatives, highly lipophilic, so better retained within the 
epidural space, short half-life, so less circulating blood levels resulting from absorption.

The present study is a prospective randomized controlled clinical comparative study done to assess the efficacy and safety of epidural butorphanol, epidural fentanyl and epidural nalbuphine, each combined with $0.125 \%$ bupivacaine for the management of post-operative pain. Total 90 patients belonging to age groups 19-65 years were taken, out of which majority of patients belonged to 20-50 years of age. The patients undergoing elective lower abdominal surgeries in general surgery, urology, gynaecology and plastic surgery were selected.

The observations of the study were analysed, and results revealed that faster onset of analgesia in fentanyl group compared to other two groups. This could be correlated with the studies conducted by Kaur et al. ${ }^{[5]}$ who studied epidural butorphanol and fentanyl as adjuvants in lower abdominal surgeries demonstrated earlier onset with fentanyl when used with bupivacaine epidurally (mean 10.80 minutes) than with butorphanol used with bupivacaine epidurally (mean 11.08 minutes).

Regarding the duration of analgesia, the duration was significantly longer in butorphanol group. The above observation correlates with study of Abboud et al. ${ }^{[6]}$ who noted the duration of analgesia to be $4.82 \pm 0.77 \mathrm{hrs}, 5.53 \pm 0.86 \mathrm{hrs}$, $8.05 \pm 0.97 \mathrm{hrs}$ after use of the first dose of $1 \mathrm{mg}, 2$ $\mathrm{mg}$, and $4 \mathrm{mg}$ butorphanol administered epidurally.Mok and Tsai ${ }^{[10]}$ who did a study to evaluate the analgesic efficacy and safety of epidural butorphanol (4 mg) in comparison to thatof epidural morphine $5 \mathrm{mg}$ in patients with post-operative pain. Intheir study, it was observed that the onset of pain relief with epidural butorphanol appeared at 15 minutes and peaked at 30 minutes. Malik et al ${ }^{\text {[4] }}$ who used $2 \mathrm{mg}$ butorphanol epidurally for post-operative analgesia after orthopedic surgeries, found duration to be $5.59 \pm 1.15$ hrs after the first dose. Kaur et $\mathrm{al}^{[6]}$ noted the duration of epidural fentanyl $100 \mu \mathrm{g}$ with $20 \mathrm{ml}$ bupivacaine was 3-9 hrs, mean duration being 5.96 hrs. Chatrath et al. ${ }^{[7]}$ used $10 \mathrm{mg}$ epidural nalbuphine along with $0.25 \%$ bupivacaine and found the duration to be $380 \pm 11.4$ minutes after lower limb and hip surgeries. From the above studies, it was demonstrated that the duration was significantly greater in butorphanol group with a mean duration of $7.64 \mathrm{hrs}$.

Side effects of opioids include nausea, vomiting, sedation, pruritus, urinary retention, and respiratory depression. Pruritus was seen in 3 patients belonging to fentanyl group. Supporting to our study, Sedation was observed in butorphanol group consistent with the study of Venkatraman et al. ${ }^{[2]}$ who observed sedation in patients receiving epidural butorphanol. This is in accordance with findings of Abboud et al ${ }^{[6]}$ who found paucity of side effects with epidural butorphanol given after cesarean section and attributed this to high lipid solubility of butorphanol thus limiting its cephalic spread to the brainstem. Chatrath et al. ${ }^{[7]}$ studied the effects of epidural nalbuphine and tramadol for postoperative analgesia in orthopaedic surgeries and concluded that patients were more comfortable after nalbuphine epidurally since they complained of lesser side effects.

\section{Conclusion}

Opioid analgesics with local anesthetics are extremely safe, effective and reliable method of post-operative pain relief. Butorphanol administered, epidurally has advantage of longer duration of analgesia than fentanyl or epidural nalbuphine with side effects like nausea vomiting and sedation. The addition of fentanyl results in faster onset of analgesia with ocassional adverse effects like pruritus than butorphanol and nalbuphine when given epidurally along with $0.125 \%$ bupivacaine. None of the patients in this study developed respiratory depression, but it is strongly recommended in concurrence with other authors that monitoring for clinical respiratory depression be made in all patients during the period of analgesia. 


\section{References}

1. Camann WR, Hurley RH, Gilbertson LI, Long ML, Datta S. Epiduralnalbuphine for analgesia following caesarean delivery: Dose-response and effect of local anaesthetic choice. Can J Anaesth 1991;38(6):728-32.

2. Venkatraman R, Sandhiya R. Evaluation of efficacy of epidural butorphanol tartarate for post-operative analgesia. Int $\mathbf{J}$ Pharm Pharm Sci 2015;7(2):52-4.

3. Hurley RH, Camann WR, B Gilbertson LI, Long ML, Datta S. Epiduralnalbuphine for analgesia following caesarean delivery: Dose-response and effect of local anaesthetic choice. Can J Anaesth 1991;38(6):728-32.

4. Malik P,Malhotra N,Manchanda C, . Comparitive evaluation of epidural fentanyl and butorphanol for postoperative analgesia. $\mathrm{J}$ Anesthesiol Clin Pharmacol 2006;22:377-82.

5. Kaur J, Bajwa SJ. Comparison of epidural butorphanol and fentanyl as adjuvants in the lower abdominal surgery: A randomized clinical study. Saudi J Anaesth 2014;8(2):167-71.

6. Abboud TK, , Zhu J,Moore M,Minehart M,Murakawa K, Longhitano M, et al. Epidural butorphanol or morphine for the relief of postcesarean section pain Ventilatory responses to carbon dioxide. Anaesth Analg 1987;66(9):887-93.

7. Chatrath V, Kaur S, Attri JP. Epidural nalbuphine for post-operative analgesia in orthopaedic surgery. Anesth Essays Res 2015;9(3):326-30.

8. Naulty JS, Weintraub S, Mcmahon J, Hunt OC, Chantigian R. Epidural butorphanol for postcesarean delivery pain management. Anesthesiology 1984; 61:A415.

9. Kim DH, Kim TJ, Park NH. Comparison of butorphanol and fentanyl administered with bupivacaine for patient controlled epidural analgesia after cesarean section. Korean J Anesthesiol 1998;34(1):126-31.

10. Mok MS, Tsai YJ, Ho WM. Efficacy of epidural butorphanol compare to morphine for the relief of post-operative pain. Anaesthesiology 1986;65:A175.

11. Parker RK, Holtmann B, White PF. Patient-controlled epidural analgesia: Interactions between nalbuphine and hydromorphone. Anesth Analg 1997;84(4):757-63.

12. Bakhshi RG, Bobby NK, Jagtap SR. Comparative study of intrathecal fentanyl versus intrathecal clonidine with low dose bupivacaine for lower abdominal and lower limb surgery. ISSP J 2009;

13. Baxter $\mathrm{AD}$, Langanière $\mathrm{S}$, Samson $\mathrm{B}$, McGilveray IJ, Hull K. A dose-response study of nalbuphine for post-thoracotomy epidural analgesia. Can J Anaesth 1991;38(2):175-82.

14. Palacios QT, Jones MM, Hawkins JL, Adenwala JN, Longmire S, Hess KR, et al. Postcesarean section analgesia: A comparison of epidural butorphanol and morphine. Can J Anaesth 1991;38(1):2430.

15. Lippmann M, Mok MS. Epidural butorphanol for the relief of postoperative pain. Anesth Analg 1988;67(4):418.

16. Hunt CO, Naulty JS, Malinow AM, Datta S, Ostheimer GW. Epidural butorphanolbupivacaine for analgesia during labor and delivery. Anesth Analg 1989;68(3):323-7. 